\title{
Nonlinear Optimal Tracking Control of a Piezoelectric Nanopositioning Stage
}

\author{
William S. Oates ${ }^{1}$ and Ralph C. Smith ${ }^{2}$ \\ Center for Research in Scientific Computation \\ Department of Mathematics \\ North Carolina State University \\ Raleigh, NC 27695
}

\begin{abstract}
High performance nanopositioning stages, used in a variety of applications such as atomic force microscopy and three-dimensional nanometer-scale lithography, require stringent position control over relatively large displacements and a broad frequency range. Piezoelectric materials, which are typically employed in nanopositioining stages, provide excellent position control when driven at relatively low frequency and low field levels. However, in applications where the stage operates over a relatively large region (microns to millimeters) and broad frequency range $(\mathrm{Hz}-\mathrm{kHz})$, piezoelectric materials often exhibit nonlinear and rate-dependent hystereis which requires control designs that can effectively accommodate such behavior. In this paper, a nonlinear, thermal-relaxation, piezoelectric constitutive law is incorporated into an open loop optimal tracking control design to accurately track a desired reference signal when nonlinearities, thermal relaxation and hyteresis are present. A comparison between linear optimal control and the nonlinear optimal control design is given to illustrate performance enhancements when the constitutive behavior is included in the control design.
\end{abstract}

Keywords: Hysteresis, piezoelectric, nonlinear control, optimal control, nanopositioning

\section{Introduction}

Highly accurate nanopositioning control becomes increasingly important in applications such image steering devices [24], piezoresponse force microscopy [5], atomic manipulation [22], and protein delivery [23]. Often, piezoelectric materials are chosen as actuators in nanopositioning stages as well as embedded actuators within MEMS devices [24]. These materials provide small displacements and large forces over a relatively large bandwith $(\mathrm{Hz}-\mathrm{MHz})$; however, nonlinearities and hysteresis often occur at moderate to large electric field levels. This behavior requires the development of a control design that ensures adequate control input is available to meet the performance objectives while accommodating constitutive nonlinearities and hysteresis. For example, the realtime monitoring of protein unfolding using an atomic force microscope requires highly accurate position control at high scan rates which are sufficient to introduce constitutive nonlinearities and hysteresis in the piezoelectric actuator and subsequently in the positioning stage. In addition, low scan rates or static displacement control can lead to drift from relaxation mechanisms thus reducing accuracy in characterizing a specimen.

In applications where high performance and accuracy are not critical, constitutive nonlinearities and hysteresis can be compensated using classical Proportional-Integral (PI) or Proportional-Integral-Derivative (PID) control, but this can potentially lead to bandwidth limitation and inefficiencies. Alternatively, it is illustrated in $[7,8]$ that the use of charge- or current-controlled amplifiers can essentially eliminate hysteresis. Unfortunately, this mode of operation can be prohibitively expensive relative to more commonly employed voltage-controlled amplifiers. Furthermore, current control is ineffective if maintaining DC offsets as is the case when the x-stage of an AFM is in a fixed position while a sweep is performed with the y-stage. The present analysis focuses on control development for these applications where nanoscale position accuracy is paramount and charge control is infeasible.

\footnotetext{
${ }^{1}$ Email: wsoates@ncsu.edu, Telephone: (919) 515-2386

22Email: rsmith@eos.ncsu.edu, Telephone: (919) 515-7552
} 
Considerable attention has been focused on appropriate control strategies to compensate for ferroelectric hysteresis and nonlinearities in piezoelectric actuators used in nanopositioning stages [3, 12, 13, 14, 19, 25]. PI and PID control designs have been shown to be limited in bandwidth and are only effective in small scanning regimes where hysteresis is minimal. At moderate to large field levels where the nonlinearties and hysteresis increase, instabilities may occur as the control gains are necessarily increased to achieve the required accuracy. The application of linear robust control techniques such as $\mathcal{H}_{\infty}$ control have provided improvements in bandwidth and robustness to nonlinearities and hysteresis $[12,13,14]$. However, when linear robust control methods are implemented, the control input focuses on compensating for the ferroelectric constitutive behavior which reduces the ability to compensate for external disturbance loads and system dynamics of the device. This has led to research focused on the design and implemention of nonlinear, model-based control designs to accommodate constitutive nonlinearities and hysteresis $[3,11,19]$.

The development and implementation of the nonlinear control design is presented as follows. In Section 2 a ferroelectric homogenized energy model is summarized and employed in a set of partial differential equations (PDE) and subsequent finite element model of the piezoelectric nanopositioning stage. In Section 3, the nonlinear control design is developed. First, open loop linear tracking control design is developed and compared to the nonlinear optimal control design.

\section{Nanopositioning Stage and Hysteresis Model}

The control design is focused on nanopositioning stages driven by piezoelectric stack actuators for applications in atomic force microscopy (AFM). The typical AFM system is illustrated in Figure 1. The side view illustrates the nanopositioning stage and piezoelectric actuator that controls specimen height relative to the cantilever. In this mode of operation, a photodiode measures changes in height of the cantilever position by reflecting a laser beam off the cantilever and a feedback law is used to reposition the specimen to maintain constant surfaces. The surface is scanned in this manner which yields the surface structure of the specimen. In-plane position control is achieved using two additional stack actuators as illustrated in the top view of the positioning stage in Figure 1(ii). In-plane actuator coupling is typically negligible in this design which allows development of the control design for a single actuator. Piezoelectric tube actuators can also be employed to control in-plane and out-of-plane displacement. Tube designs have provided improved linear behavior but the non-negligible coupling between in-plane displacements further complicates the control design [19]. The control design presented here is suitable for accommodating coupling present in a piezoelectric-tube actuator, but the finite element implementation is more complex; e.g., see [17]. The focus here is on control development, therefore the decoupled stack actuator nanopositioner design is considered.

The model development employed in the present analysis focuses on a homogenized energy framework to formulate a highly accurate displacement control that can be efficiently implemented in real-time applications. The constitutive model is based on a previously developed homogenized energy-based model [16, 18, 20, 21]. The model incorporates mesocopic material behavior at the domain or grain level in a stochastic homogenization framework to predict macroscopic material behavior. A distribution of interaction fields and coercive fields are implemented to model polarization switching processes that typically occur in the presence of material inhomogeneities and residual fields. Boltzmann relations are included to model thermal relaxation behavior when thermal energy affects polarization switching. Macroscopic material behavior is determined by homogenizing the local polarization variants according to the distribution of interaction and coercive fields.

\subsection{Homogenized Energy Model}

The equations governing the homogenized energy model are summarized here. A detailed review of the modeling framework is given in $[16,18,20]$. The constitutive law is focused on uniaxial loading of rod-type actuators since the decoupled piezoelectric stack actuators are assumed in the control design of the nanopositioning stage for in-plane position control. The Gibbs energy at the mesoscopic length scale is

$$
G(P, T)=\Psi(P, T)-E P
$$

where $\Psi(P, T)$ is the Helmholtz energy detailed in [16], $T$ is temperature, $E$ is the electric field, and $P$ is the polarization. In the one-dimensional case considered here, the Helmholtz energy function is a double-well 


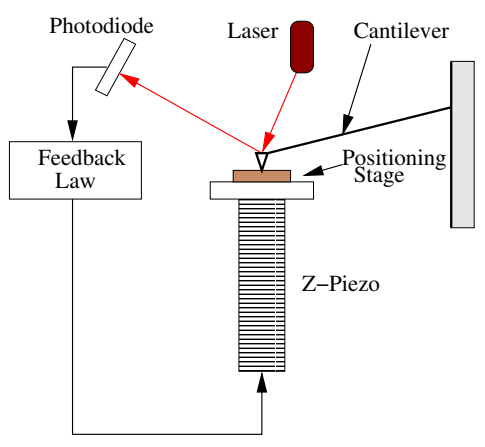

(i)

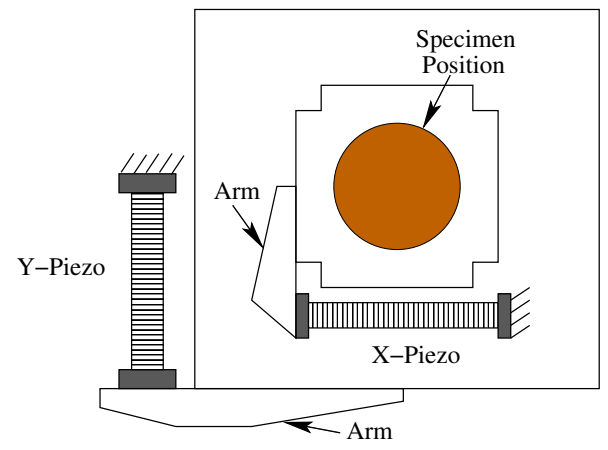

(ii)

Figure 1: Schematic of an atomic force microscope configuration used in constructing the nonlinear control design. (i) Side view of the AFM set-up with out-of-plane displacement control piezoelectric actuator. (ii) Top view of the positioning stage illustrating the configuration of the in-plane displacement control piezoelectric actuators.

potential below the Curie point $T_{c}$ which gives rise to stable spontaneous polarization with equal magnitude in the positive and negative directions.

Thermal relaxation in the piezoelectric material is often present and must be addressed in the constitutive model to predict creep that may occur in the nanopositioning stage. This can be accomplished by employing the Boltzmann relation

$$
\mu(G)=C e^{-G V / k T}
$$

which quantifies the probability $\mu$ of achieving an energy level $G$. The relative thermal energy $(k T / V)$ is defined over a representative volume element $V$ at the mesoscopic length scale, $k$ is Boltzmann's constant, and the constant $C$ is specified to ensure integration to unity. The inclusion of thermal energy in the energy function incorporates the effect of switching prior to the minima in G disappearing as the temperature increases. This reduces the sharp transition of ferroelectric switching near the coercive field as the thermal energy increases.

The Boltzmann relation gives rise to the expected values

$$
\left\langle P_{+}\right\rangle=\int_{P_{I}}^{\infty} P \mu(G) d P \quad, \quad\left\langle P_{-}\right\rangle=\int_{-\infty}^{-P_{I}} P \mu(G) d P
$$

of the polarization associated with positively and negatively oriented variants. Here $\pm P_{I}$ are the positive and negative inflection points in the Helmholtz energy definition. Kinetic equations are developed that define the volume fraction of positive $x_{+}$and negative polarization variants $x_{-}$. The kinetic equations rely on transition likelihoods that define the probability that polarization variants switch according to the energy relations; see $[16,18,21]$ for details.

The resulting local average polarization is quantified by the relation

$$
\bar{P}=x_{+}\left\langle P_{+}\right\rangle+x_{-}\left\langle P_{-}\right\rangle .
$$

The macroscopic polarization is computed from the distribution of local variants from the relation

$$
[P(E)](t)=\int_{-\infty}^{\infty} \int_{0}^{\infty} \nu\left(E_{c}, E_{I}\right)\left[\bar{P}\left(E+E_{I} ; E_{c}, \xi\right)\right](t) d E_{I} d E_{c}
$$


where $\nu\left(E_{c}, E_{I}\right)$ denotes the distribution of coercive and interaction fields and $\xi$ represents the initial distribution of the local variants. The local polarization $\bar{P}\left(E+E_{I} ; E_{c}, \xi\right)$ is determined from (4) where the dependence on interaction fields and coercived fields is embedded in the kinetic equations that describe $x_{+}$and $x_{-}$. The density $\nu\left(E_{c}, E_{I}\right)$ is chosen as

$$
\nu\left(E_{c}, E_{I}\right)=c_{1} e^{-\left[\ln \left(E_{c} / \bar{E}_{c}\right) / 2 c\right]^{2}} e^{-E_{I}^{2} / 2 b^{2}}
$$

where $\bar{E}_{c}$ is the average coercive field, $c$ quantifies the coercive field variability, $b$ is the variance of the interaction field, and $c_{1}$ is a scaling parameter. Techniques to identify general densities can be found in [18]. Numerical techniques to approximate (5) and comparison to experimental results can be found in [16].

Whereas the macroscopic polarization is quantified by (5), the forces generated by the stack actuator must be quantified for implementation within the control design. This is provided by the constitutive law

$$
\sigma=Y^{P} \varepsilon+c_{D} \dot{\varepsilon}-h_{1}\left(P(E)-P^{r}\right)-h_{2}\left(P(E)-P^{r}\right)^{2}
$$

representing uniaxial stress in the piezoelectric stack actuator where the effective properties of the actuator include $Y^{P}$ as the elastic modulus at constant polarization, $c_{D}$ as the Kelvin-Voigt damping parameter, $\varepsilon$ as the linear strain component in the direction of loading, $h_{1}$ as the piezoelectric coefficient and $h_{2}$ as the electrostrictive coefficient. It is assumed that stress fields are limited to the linear elastic regime where ferroelastic switching (i.e., stress induced domain switching) is negligible. The polarization $P(E)$ is computed using (5) where $P^{r}$ is the initial macroscopic remanent state of the material. In the simulations presented, the actuator model is intially poled with a field equal to $2 \bar{E}_{c}$ which results in $P^{r}=0.206 \mathrm{C} / \mathrm{m}^{2}$.

Simulations of minor loop hysteresis are illustrated in Figure 2 for the case of zero applied stress. Model predictions illustrate drift in the polarization and strain behavior for an input sinusoidal waveform at $1 \mathrm{~Hz}$. The material parameters associated with the homogenized energy model are given in Table 1 . The size of the representative volume element $V$ was selected to give typical relaxation behavior for an isothermal process and internal damping behavior at room temperature.

The stress computed using (7) includes linear stress-strain behavior as well as nonlinear and hysteretic dependence on the electric field through the $P(E)$ relation. It does not include spatial dependence. This is incorporated in the structural model in the following section.

\subsection{Structural Model}

The constitutive relations (5) and (7) are used to develop a system model that quantifies forces and displacements in the presence of applied fields and stress that occur during operation of the nanopositioning stage. The partial differential equation (PDE) model is first given and then formulated as set of ordinary differential equations (ODEs) through a finite element discretization in space. The structural coupling of the nanopositioning stage is modeled as a damped oscillator to account for boundary conditions at the end of the piezoelectric actuator. The geometry used in constructing the structural model is illustrated in Figure 3.

The equation of motion for the structural model is given by the relation $[4,16]$

Table 1: Parameters employed in the homogenized energy model.

$$
\begin{aligned}
& c_{1}=0.54 C / M V^{2} \\
& c=0.4 \\
& b=0.87 \mathrm{MV} / \mathrm{m} \\
& \chi_{e}=0.008 \mathrm{C} / \mathrm{mV} \\
& T=300 \mathrm{~K} \\
& V=1.25 \times 10^{5} \mathrm{~nm}^{3} \\
& \tau=100 \mathrm{~ns} \\
& \hline
\end{aligned}
$$




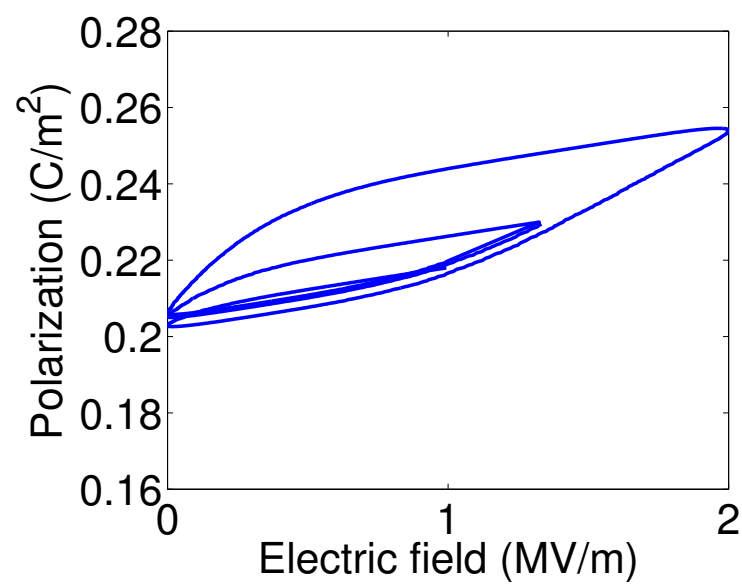

(a)

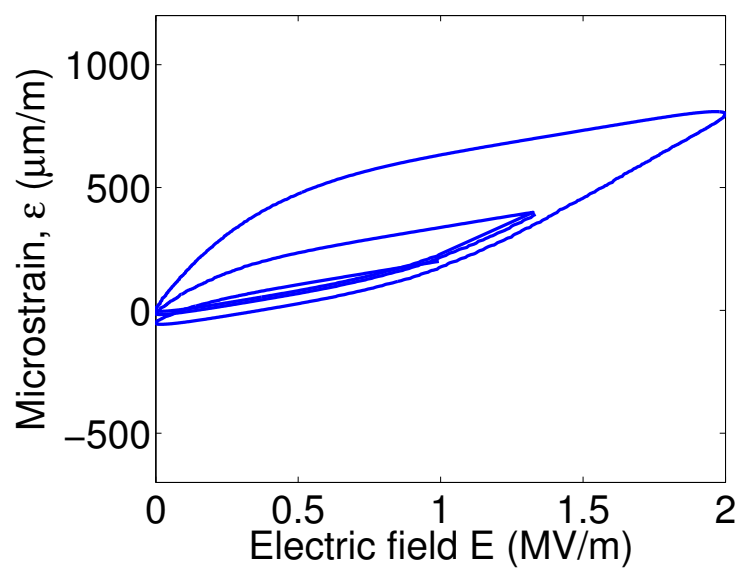

(b)

Figure 2: Nonlinear and hysteretic homogenized energy model results for minor loop hysteresis typically observed in ferroelectric materials under zero stress. (a) Electric field versus polarization. (b) Electric field versus longitudinal microstrain.

$$
\rho A \frac{\partial^{2} w}{\partial t^{2}}=\frac{\partial N_{t o t}}{\partial x}
$$

where the mass density of the actuator is denoted by $\rho$, the cross-section area is $A$ and the displacement is denoted by $w$. The total force $N_{t o t}$ acting on the actuator is

$$
N_{t o t}(t, x)=Y^{P} A \frac{\partial w}{\partial x}+c_{D} A \frac{\partial^{2} w}{\partial x \partial t}+F_{p}(E)+F_{d}
$$

where the the first term on the right hand side of the equation represents the elastic restoring force and a linear damping coefficient is incorporated in the second term. The linear elastic strain component in the direction of loading is defined by $\varepsilon=\frac{\partial w}{\partial x}$. The term $F_{d}$ incorporates external disturbance loads and the coupling force $F_{p}$ represents forces generated by an applied electric field where

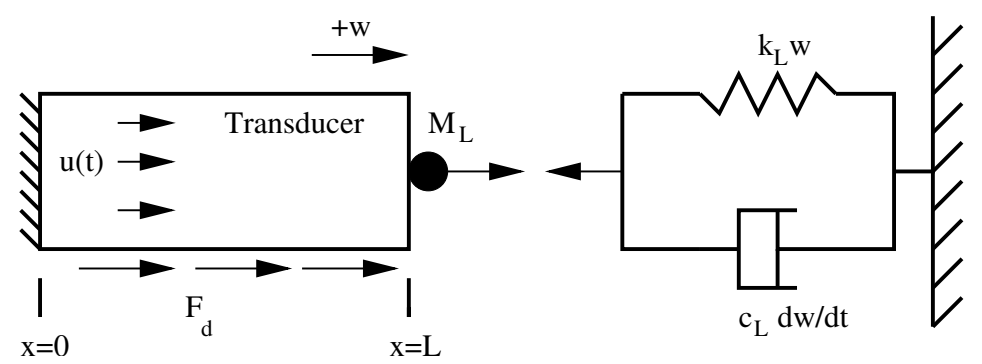

Figure 3: Piezoelectric stack actuator with damped oscillator used to quantify loads during scanning operations of an AFM nanopositioning stage. Disturbance forces along the actuator are given by $F_{d}$ and the control input is $u(t)$. 
Table 2: Model parameters for the piezoelectric stack actuator and damped oscillator.

\begin{tabular}{l|l|l}
\hline$Y^{P}=60 \times 10^{9} \mathrm{~N} / \mathrm{m}^{2}$ & $\rho=7.5 \times 10^{3} \mathrm{~kg} / \mathrm{m}^{3}$ & $k_{L}=3.04 \times 10^{6} \mathrm{~N} / \mathrm{m}$ \\
$h_{1}=1.0 \times 10^{6} \mathrm{~N} / \mathrm{C}$ & $A=5.07 \times 10^{-4} \mathrm{~m}^{2}$ & $c_{L}=3.04 \times 10^{2} \mathrm{Ns} / \mathrm{m}$ \\
$h_{2}=1.0 \times 10^{3} \mathrm{Nm}^{2} / \mathrm{C}^{2}$ & $c_{D}=3.7 \times 10^{6} \mathrm{Ns} / \mathrm{m}$ & $m_{L}=40 \mathrm{~g}$ \\
$P_{R}=0.3 \mathrm{C} / \mathrm{m}^{2}$ & $L=0.1 \mathrm{~m}$ & \\
\hline
\end{tabular}

$$
F_{p}(E)=A\left[h_{1}\left(P(E)-P^{r}\right)+h_{2}\left(P(E)-P^{r}\right)^{2}\right]
$$

and the hysteretic and nonlinear $E-P$ relation is specified by (5).

As illustrated in Figure 3, the boundary conditions are defined by a zero displacement at $x=0$ and the balance of forces between the piezoelectric actuator and the damped oscillator at $x=L$ yields

$$
N_{t o t}(t, L)=-k_{L} w(t, L)-c_{L} \frac{\partial w}{\partial t}(t, L)-m_{L} \frac{\partial^{2} w}{\partial t^{2}}(t, L) .
$$

The initial conditions are $w(0, x)=0$ and $\frac{\partial w}{\partial x}(0, x)=0$. Model parameters associated with the stack actuator and damped oscillator used in the control design are given in Table 2.

The strong form of the PDE model given by (8) can be written in the weak form for finite element implementation; see [16] for details. The weak form of the model given is used to obtain a matrix ODE system given by

$$
\mathbf{M} \ddot{\mathbf{w}}+\mathbf{C}_{D} \dot{\mathbf{w}}+\mathbf{K} \mathbf{w}=F_{p}(E) \mathbf{b}+\mathbf{f}_{\mathbf{d}}
$$

where $\mathbf{w}(t)=\left[w_{1}(t), \ldots, w_{N}(t)\right]$ are the nodal solutions obtained from the weak formulation and finite element discretization. $\mathbf{M} \in \mathbb{R}^{N \times N}, \mathbf{C}_{D} \in \mathbb{R}^{N \times N}$ and $\mathbf{K} \in \mathbb{R}^{N \times N}$ denote the mass, damping and stiffness matrices. The vectors $\mathbf{b} \in \mathbb{R}^{N}$ and $\mathbf{f}_{\mathbf{d}} \in \mathbb{R}^{N}$ include the integrated basis functions related to the control input and disturbance loads, respectively. Components contained within the system matrices and vectors can be found in $[11,16]$.

Formulation of (12) as a first order system yields

$$
\begin{aligned}
& \dot{\mathbf{x}}(t)=\mathbf{A} \mathbf{x}(t)+[\mathbf{B}(u)](t)+\mathbf{G}(t) \\
& \mathbf{x}(0)=\mathbf{x}_{\mathbf{0}} \\
& y(t)=\mathbf{C x}(t)
\end{aligned}
$$

where $\mathbf{x}(t)=[\vec{w}, \dot{\vec{w}}]^{T}$ should not to be confused with the coordinate $x$. The matrix $\mathbf{A}$ incorporates the mass, damping and stiffness matrices given in (12) and $[\mathbf{B}(u)](t)$ includes the nonlinear input where $u(t)$ is defined as the electric field. The initial conditions are defined by $\mathbf{x}_{\mathbf{0}}$. The output of the system $y(t)$ is a function of the system states according to the matrix $\mathbf{C}$. In the nanopositioning stage, it is assumed that only the displacement at $x=L$ is observable which results in $\mathbf{C}=\left[\begin{array}{llll}1 & 0 & \ldots & 0\end{array}\right]$ with dimension $1 \times 2 N$. External disturbances are incorporated in $\mathbf{G}$.

A temporal discretization of the system given by (13) is used to numerically analyze the dynamic performance. The trapezoid rule is adopted since it is moderately accurate, A-stable, and requires minimal computer storage capacity. The trapezoidal discretization yields the relation for each iteration

$$
\begin{aligned}
& \mathbf{x}_{k+1}=\mathbf{W} \mathbf{x}_{k}+\mathbf{V}\left[\mathbf{B}\left(u_{k}\right)\right]+\frac{1}{2} \mathbf{V}\left[\mathbf{G}\left(t_{k}\right)+\mathbf{G}\left(t_{k+1}\right)\right] \\
& \mathbf{x}(0)=\mathbf{x}_{\mathbf{0}}
\end{aligned}
$$


where a temporal step size $\Delta t$ is employed giving a discretization in time defined by $t_{k}=k \Delta t$. The values $\mathbf{x}_{k}$ approximate $\mathbf{x}\left(t_{k}\right)$. The matrices

$$
\begin{aligned}
& \mathbf{W}=\left[\mathbf{I}-\frac{\Delta t}{2} \mathbf{A}\right]^{-1}\left[\mathbf{I}+\frac{\Delta t}{2} \mathbf{A}\right] \\
& \mathbf{V}=\Delta t\left[\mathbf{I}-\frac{\Delta t}{2} \mathbf{A}\right]^{-1}
\end{aligned}
$$

are created once for numerical implementation yielding approximate solutions with $O\left(h^{2},(\Delta t)^{2}\right)$ accuracy. In (14), only values for the control input at the present time $t_{k}$ are included in the temporal discretization to model dynamics of real-time feedback developed in the following section. This approach is employed since the control input can only be dependent on the current and previous states.

\section{Control Design}

We compare the performance between linear and nonlinear optimal control designs when ferroelectric nonlinearities, hysteresis and relaxation behavior are present in the piezoelectric actuator. The ferroelectric constitutive behavior is shown to introduce significant degradation in position accuracy when linear optimal control methods are employed. Improvements in high accuracy tracking are then obtained by implementing a nonlinear optimal control design. Actuator displacements on the order of tens of microns are chosen for the analysis where it has been demonstrated that nonlinearities and hysteresis are significant. The reference tracking profile used in the simulations is based on a typical scan and hold procedure used in nano- and micron-scale materal characterization. This procedure requires that the specimen translate in the $x$-direction while holding in the $y$-direction. At the end of each scan, the position is held in the $x$-direction and increased or decreased a small amount in the $y$-direction and the procedure is repeated in the negative $x$-direction. A low frequency scan rate is chose for the simulations $(0.2 \mathrm{~Hz})$ where relaxation and creep are significant.

\subsection{Optimal Tracking Control}

Development of the nonlinear optimal tracking control design follows a previous approach focused on negligible relaxation hysteresis of magnetostrictive actuators for vibration attenuation of beam and plate structures and tracking control of rod structures $[10,11,15]$. We summarize here key equations associated with optimal tracking control and its application in compensating ferroelectric nonlinearities, hysteresis and relaxation behavior.

Optimal tracking control utilizes a cost functional to determine the optimal control input. The cost functional

$$
\bar{J}=\frac{1}{2}\left(\mathbf{C x}\left(t_{f}\right)-r\left(t_{f}\right)\right)^{T} P\left(\mathbf{C x}\left(t_{f}\right)-r\left(t_{f}\right)\right)+\int_{t_{0}}^{t_{f}}\left[H-\boldsymbol{\lambda}^{T}(t) \dot{\mathbf{x}}(t)\right] d t
$$

penalizes the control input and the error between the piezoelectric actuator displacement and the prescibed displacement where $P$ penalizes large terminal values on the tracking error, $H$ is the Hamiltonian, and $\boldsymbol{\lambda}(t)$ is a set of Lagrange multipliers. The Hamiltonian is

$$
\begin{aligned}
H= & \frac{1}{2}\left[(\mathbf{C x}(t)-r(t))^{T} Q(\mathbf{C x}(t)-r(t))+u^{T}(t) R u(t)\right] \\
& +\boldsymbol{\lambda}^{T}[\mathbf{A x}(t)+[\mathbf{B}(u)](t)+\mathbf{G}(t)]
\end{aligned}
$$

where penalties on the tracking error and the control input are given by the variables $Q$ and $R$, respectively.

The minimum of the cost functional in (16) is determined under the constraint of the differential equation given by (13). By employing Lagrange multipliers an unconstrained minimization problem is constructed where the stationary condition for the Hamiltonian yields the adjoint relation $[2,6]$ 


$$
\dot{\boldsymbol{\lambda}}(t)=-\mathbf{A}^{\mathbf{T}} \boldsymbol{\lambda}(t)-\mathbf{C}^{\mathbf{T}} Q \mathbf{C x}(t)+\mathbf{C}^{\mathbf{T}} Q r(t)
$$

and optimal control input

$$
u^{*}(t)=-R^{-1}\left(\frac{\partial \mathbf{B}(u)}{\partial u}\right)^{T} \boldsymbol{\lambda}(t) .
$$

The resulting optimality system is

$$
\begin{aligned}
& {\left[\begin{array}{c}
\dot{\mathbf{x}}(t) \\
\dot{\boldsymbol{\lambda}}(t)
\end{array}\right]=\left[\begin{array}{l}
\mathbf{A} \mathbf{x}(t)+[\mathbf{B}(u)](t)+\mathbf{G}(t) \\
-\mathbf{A}^{\mathbf{T}} \boldsymbol{\lambda}(t)-\mathbf{C}^{\mathbf{T}} Q \mathbf{C x}(t)+\mathbf{C}^{\mathbf{T}} Q r(t)
\end{array}\right]} \\
& \mathbf{x}\left(t_{0}\right)=\mathbf{x}_{\mathbf{0}} \\
& \boldsymbol{\lambda}\left(t_{f}\right)=\mathbf{C}^{\mathbf{T}} P\left(\mathbf{C x}\left(t_{f}\right)-r\left(t_{f}\right)\right) .
\end{aligned}
$$

The force determined from (10) is included in the input operator $[\mathbf{B}(u)](t)$ which directly includes the nonlinear and hysteretic $E-P$ relation as well as relaxation behavior within the control formulation. This system of equations results in a two-point boundary value problem which presents challenges in obtaining a solution for large systems. Furthermore, the nonlinear nature of the input operator precludes an efficient Riccati formulation.

\subsubsection{Linear Optimal Control}

If the input operator is assumed to be linear and not a function of the polarization state (i.e. valid for small to moderate field inputs), the control design can be simplified by determining the suboptimal control input from the algebraic Riccati equation

$$
\mathbf{A}^{T} \boldsymbol{\Pi}+\boldsymbol{\Pi} \mathbf{A}-\boldsymbol{\Pi B} R^{-1} \mathbf{B}^{T} \boldsymbol{\Pi}+\mathbf{C}^{T} Q \mathbf{C}=0
$$

see $[2,6]$ for details.

The linear optimal control input is then defined by

$$
u^{*}(t)=-R^{-1} \mathbf{B}^{T}[\boldsymbol{\Pi} \mathbf{x}(t)-\boldsymbol{\nu}(t)]
$$

where the variable $\boldsymbol{\nu}(t) \in \mathbb{R}^{2 N}$ is the solution to the auxillary differential equation

$$
\begin{aligned}
& \dot{\boldsymbol{\nu}}(t)=-\left[\mathbf{A}-\mathbf{B} R^{-1} \mathbf{B}^{T} \boldsymbol{\Pi}\right]^{T} \boldsymbol{\nu}(t)-\mathbf{C}^{T} Q r(t)+\mathbf{\Pi G}(t) \\
& \boldsymbol{\nu}\left(t_{f}\right)=\mathbf{C}^{T} \mathbf{P} r\left(t_{f}\right) .
\end{aligned}
$$

\subsubsection{Nonlinear Open Loop Optimal Tracking Control}

When nonlinearities in the input operator cannot be neglected, (20) has the general form

$$
\begin{aligned}
& \dot{\mathbf{z}}(t)=\mathbf{F}(t, \mathbf{z}) \\
& \mathbf{E}_{\mathbf{0}} \mathbf{z}\left(t_{0}\right)=\left[\mathbf{x}_{\mathbf{0}}, \mathbf{0}\right]^{T} \\
& \mathbf{E}_{\mathbf{f}} \mathbf{z}\left(t_{f}\right)=[\mathbf{0}, \mathbf{0}]^{T}
\end{aligned}
$$

where $\mathbf{z}=[\mathbf{x}(t), \lambda(t)]^{T}$ and 


$$
\begin{aligned}
& \mathbf{F}(t, \mathbf{z})=\left[\begin{array}{l}
\mathbf{A x}(t)+[\mathbf{B}(u)](t)+\mathbf{G}(t) \\
-\mathbf{A}^{T} \lambda(t)-\mathbf{C}^{T} Q \mathbf{C} \mathbf{x}(t)+\mathbf{C}^{T} Q r(t)
\end{array}\right] \\
& \mathbf{E}_{\mathbf{0}}=\left[\begin{array}{ll}
\mathbf{I} & \mathbf{0} \\
\mathbf{0} & \mathbf{0}
\end{array}\right] \quad, \quad \mathbf{E}_{\mathbf{f}}=\left[\begin{array}{cc}
\mathbf{0} & \mathbf{0} \\
-\mathbf{C}^{T} P \mathbf{C} & \mathbf{I}
\end{array}\right] .
\end{aligned}
$$

Here $\mathbf{I}$ denotes an identity matrix with dimension corresponding to the number of basis functions employed in the spatial approximation of the state variables. Also note that the prescribed displacement has been restricted to $r\left(t_{f}\right)=0$.

The system given by (24) is approximated by discretizing the time interval $\left[t_{0}, t_{f}\right]$ with a uniform step size $\Delta t$ at the points $t_{0}, t_{1}, \cdots, t_{N}=t_{f}$. The approximate values of the state solutions and the adjoint at each time step are denoted by $\mathbf{z}_{\mathbf{0}}, \cdots, \mathbf{z}_{\mathbf{N}}$. Whereas several techniques are available to approximate the solution to (24), such as finite differences and nonlinear multiple shooting [1], a central difference of the temporal derivative is utilized here which provides a formulation that can be cast in a analytic $L U$ decomposition to solve the two-point boundary value problem, details describing the numerical approach are described elsewhere [11]. This approach results in expressing (24) as the problem of finding $\mathbf{z}_{\mathbf{h}}=\left[\mathbf{z}_{0}, \cdots, \mathbf{z}_{N}\right]$ which solves

$$
\mathcal{F}\left(\mathbf{z}_{\mathbf{h}}\right)=0 .
$$

Equation (26) includes the optimality system at each time step and the boundary conditions given in (25). Details are given in [15].

A quasi-Newton iteration of the form

$$
\mathbf{z}_{h}^{k+1}=\mathbf{z}_{h}^{k}+\boldsymbol{\xi}_{h}^{k},
$$

where $\boldsymbol{\xi}_{h}^{k}$ solves

$$
\mathcal{F}^{\prime}\left(\mathbf{z}_{h}^{k}\right) \xi_{h}^{k}=-\mathcal{F}\left(\mathbf{z}_{h}^{k}\right),
$$

is then used to approximate the solution to the nonlinear system given by (26).

\subsubsection{Simulation Results}

Improvements in tracking control are demonstrated here by comparing the linear and nonlinear optimal control designs. The values used to penalized the displacement error and control input were $Q=1 \times 10^{16}$ and $R=1 \times 10^{-8}$. The penalty on the final state was $P=1.4 \times 10^{10}$. In Figure 4 , linear optimal control illustrates poor tracking performance using a scan rate of $0.2 \mathrm{~Hz}$. The thermal relaxation effect is significant in regions of static displacement as seen in Figure 4(a). In Figure 5, the nonlinear control design is applied to the piezoelectric actuator again using a scan rate of $0.2 \mathrm{~Hz}$. Significant improvements are achieved in tracking the reference signal. In Figure 5(a) the commanded displacement and error between the commanded displacement and reference displacement are given. Whereas relaxation behavior is present at this operating frequency as illustrated in Figure 5(b), the nonlinear control design significantly reduces error relative to the linear control design. This is clearly demonstrated in regions of static displacement where the control input increases or decreases to ensure the polarization is constant which gives rise to static displacement.

\section{Concluding Remarks}

An open loop nonlinear optimal control design has been applied to a nanopositioning stage for quasi-static scanning rates for applications where nanoscale resolution is important. A homogenized energy model was implemented in the control design to account for local residual interaction fields and variations in the coercive field. Relaxation behavior was incorporated into the constitutive model to account for creep during quasi-static actuation. The nonlinear control design significantly improved reduction in tracking errors relative to linear 


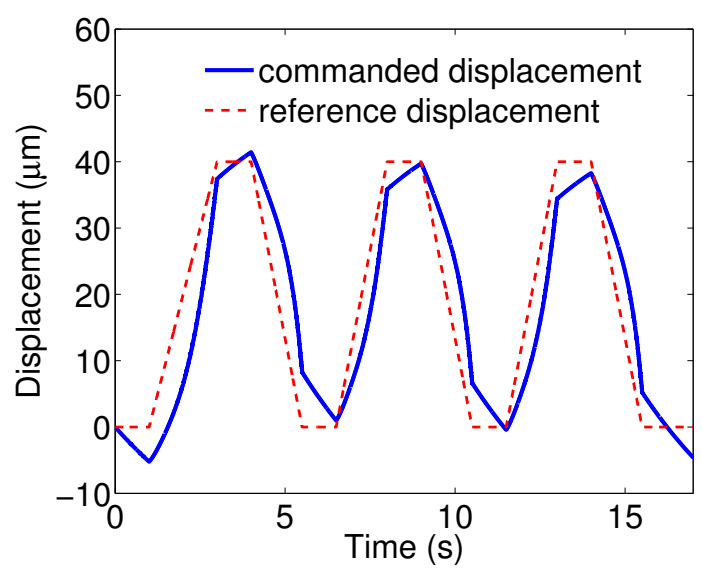

(a)

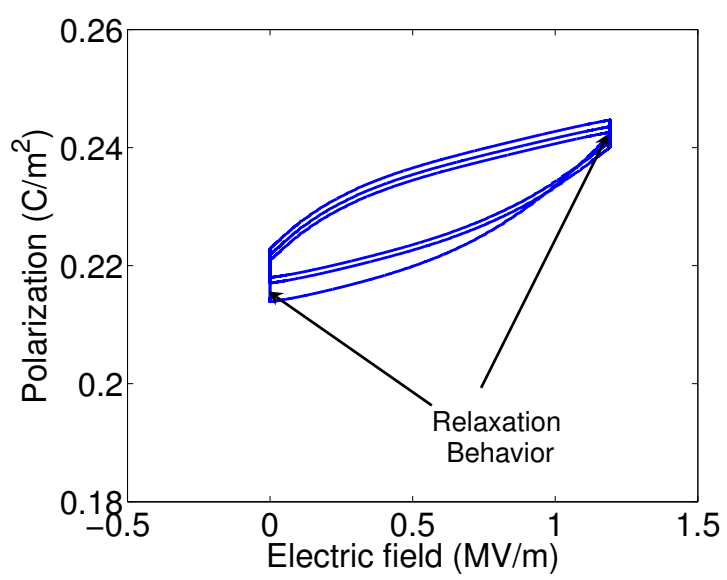

(b)

Figure 4: Tracking performance using linear optimal control to compensate for relaxation behavior at $0.2 \mathrm{~Hz}$. (a) Commanded displacement and the desired reference signal. (b) The associated control input and polarization relaxation behavior.

optimal control. However, open loop control can lead to deleterious effects when operating uncertainties are present. Improved robustness can be achieved by introducing perturbation feedback around the optimal open loop control input but this is beyond the scope of the paper. Details decribing performance robustness for tracking control using perturbation feedback can be found in $[9,11]$.

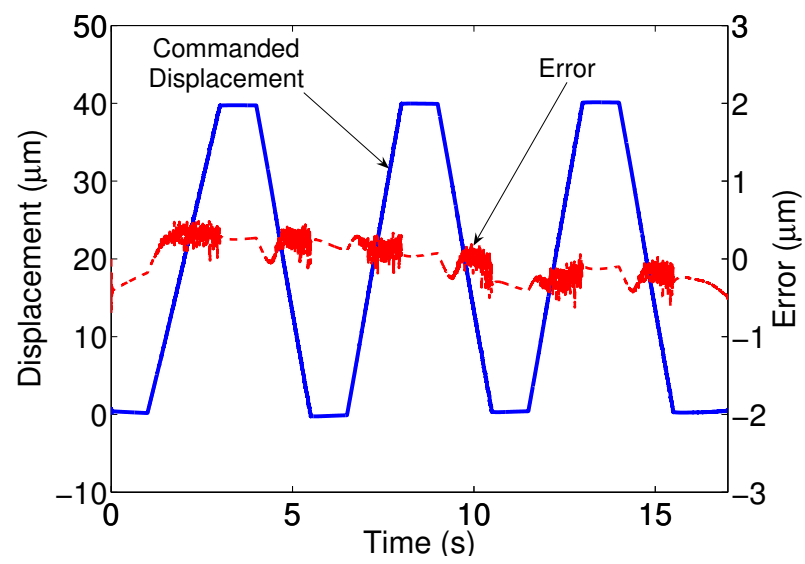

(a)

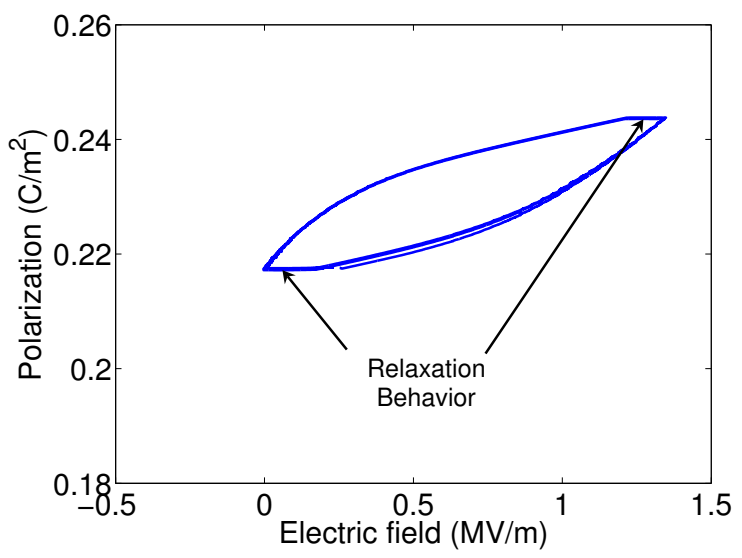

(b)

Figure 5: High performance tracking using nonlinear optimal control to compensate for relaxation behavior at $0.2 \mathrm{~Hz}$. (a) Commanded displacement and error between the actuator response and the reference signal. (b) The associated control input and polarization relaxation behavior. 


\section{Acknowledgments}

The authors gratefully acknowledge support from the Air Force Office of Scientific Research through the grant AFOSR-FA9550-04-1-0203.

\section{References}

[1] Ascher, U., Mattheij, R., and Russell, R., 1995. Numerical Solution of Boundary Value Problems for Ordinary Differential Equations. SIAM Classics in Applied Mathematics.

[2] Bryson, A., and Ho, Y.-C., 1969. Applied Optimal Control. Blasidell Publishing Company, Waltham, MA.

[3] Croft, D., and Devasia, S., 1999. "Vibration compensation for high speed scanning tunneling microscopy". Rev. Sci. Instrum., 70(12), pp. 4600-4605.

[4] Dapino, M., Smith, R., and Flatau, A., 2000. "A structural strain model for magnetostrictive transducers". IEEE T. Magn., 36(3), pp. 545-556.

[5] Gruverman, A., Cao, W., Bhaskar, S., and Dey, S., 2004. "Investigation of $\mathrm{Pb}(\mathrm{Zr}, \mathrm{Ti}) \mathrm{O}_{3} / \mathrm{GaN}_{\text {heterostruc- }}$ tures by scanning probe microscopy". Appl. Phys. Lett., 84(25), pp. 5153-5155.

[6] Lewis, F., and Syrmos, V., 1995. Optimal Control. John Wiley and Sons, New York, NY.

[7] Main, J., Garcia, E., and Newton, D., 1995. "Precision position control of piezoelectric actuators using charge feedback". J. Guid. Control Dynam., 18(5), pp. 1068-1073.

[8] Main, J., Garcia, E., Newton, D., Massengil, L., and Garcia, E., 1996. "Efficient power amplifiers for piezoelectric applications". Smart Mater Struct, 5(6), pp. 766-775.

[9] Oates, W., and Smith, R. "Nonlinear control design for a piezoelectric-driven nanopositioning stage". $J$. Dyn. Syst.-T. ASME, (CRSC technical report CRSC-TR05-43), submitted.

[10] Oates, W., and Smith, R. "Nonlinear optimal control techniques for vibration attenuation using nonlinear magnetostricive actuators". J. Intell. Mater. Sys. Struct. (CRSC technical report CRSC-TR05-25), submitted.

[11] Oates, W., and Smith, R. "Optimal tracking using magnetostricive actuators operating in the nonlinear and hysteretic regime". J. Dyn. Syst.-T. ASME, CRSC technical report CRSC-TR05-36, submitted.

[12] Salapaka, S., Sebastian, A., Cleveland, J., and Salapaka, M., 2002. "High bandwidth nano-positioner: A robust control approach". Rev. Sci. Instrum., 73(9), pp. 3232-3241.

[13] Schitter, G., Menold, P., Knapp, H., Allgower, F., and Stemmer, A., 2003. "High performance feedback for fast scanning atomic force microscopes". Rev. Sci. Instrum., 72(8), pp. 3320-3327.

[14] Sebastion, A., and Salapaka, S., 2003. "H $\mathcal{H}_{\infty}$ loop shaping design for nano-positioning". IEEE Proc. Amer. Control Conf., pp. 3708-3713.

[15] Smith, R., 1995. "A nonlinear optimal control method for magnetostrictive actuators". J. Intell. Mater. Syst. Struct., 9(6), pp. 468-486.

[16] Smith, R., 2005. Smart Material Systems: Model Development. SIAM, Philadelphia, PA.

[17] Smith, R., Hatch, A., De, T., Salapaka, M., del Rosario, R., and Raye, J. "Model development for atomic force microscope mechanisms". CRSC-TRO5-25, SIAM J. Appl. Math, submitted. 
[18] Smith, R., Hatch, A., Mukherjee, B., and Liu, S., 2005. "A homogenized energy model for hysteresis in ferroelectric materials: General density formulations". J. Intell. Mater. Syst. Struct., 16(9), pp. 713-732.

[19] Smith, R., Salapaka, M., Hatch, A., Smith, J., and De, T., 2002. "Model development and inverse compensator design for high speed nanopositioning". Proc. 41nd IEEE Conf. Decision Control, pp. 3652-3657.

[20] Smith, R., Seelecke, S., Dapino, M., and Ounaies, Z., 2005. "A unified framework for modeling hysteresis in ferroic materials". J. Mech. Phys. Solids, 54(1), pp. 46-55.

[21] Smith, R., Seelecke, S., Ounaies, Z., and Smith, J., 2003. "A free energy model for hysteresis in ferroelectric materials". J. Intell. Mater. Syst. Struct., 14(11), pp. 719-739.

[22] Sugimoto, Y., Abe, M., Hirayama, S., Oyabu, N., Custance, O., and Morita, S., 2005. "Atom inlays performed at room temperature using atomic force microscopy". Nat. Mater., 4, pp. 156-159.

[23] Tang, Q., Zhang, Y., Chen, L., Yan, F., and Wong, R., 2005. "Protein delivery with nanoscale precision". Nanotechnology, 16, pp. 1062-1068.

[24] Wong, C., Jeon, Y., Barbastathis, G., and Kim, S.-K., 2004. "Analog piezoelectric-driven tunable gratings with nanometer resolution". J. Microelectromech. S., 13(6), pp. 998-1005.

[25] Zou, Q., and Devasia, S., 2004. "Preview-based optimal inversion for output tracking: Applications to scanning tunneling microscopy". IEEE T. Contr. Syst. T., 12(3), pp. 375-386. 